\title{
Estudio Fluviomorfológico del Río Napo para la Protección de sus Orillas, Caso Parroquia de Pañacocha y sus Embarcaderos
}

Fluviomorphological Study of the Napo River for the Protection of its Banks, Case of Pañacocha Parish and its Jetties.

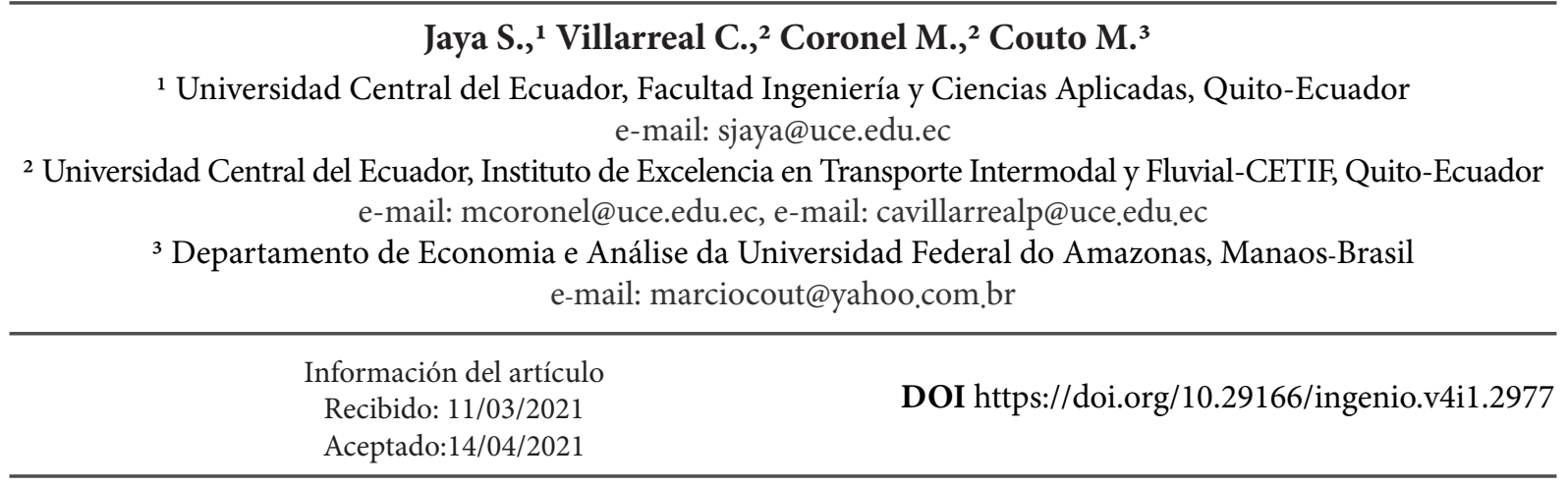

\section{RESUMEN}

Mediante estudios hidrológicos e hidráulicos-batimétricos, se determinaron las causas que producen la erosión de la ribera del río Napo-Ecuador, en la población de Pañacocha y de sus embarcaderos. Este problema se da por las olas producidas por el paso de embarcaciones a altas velocidades, crecidas súbitas, material flotante y «flujos transversales». Como solución, se analizó la construcción de obras como muros laterales, pilotajes, espigones, pero por sus costos elevados y poca efectividad observada en otros tramos del río, se descartaron. Esta investigación determinó que una buena forma de proteger este tramo, es mediante el uso de plantas nativas del sector como el Zygia Longifolia (Chiparo), cuyas raíces al ser abundantes y largas, forman una verdadera pantalla de protección contra la erosión, así se evidencia con plantas que se van sembrando año tras año. Esta investigación se realizó con el apoyo económico de ARES del Gobierno Belga.

Palabra clave: Pañacocha, erosión, río Napo, protección de márgenes, plantas nativas.

\begin{abstract}
Through hydrological and hydraulic-bathymetric studies, the causes that produce the erosion of the banks of the Napo river-Ecuador, in the town of Pañacocha and its piers, were determined. This problem occurs due to the waves produced by the passage of boats at high speeds, sudden floods, floating material and "transversal flows». As a solution, the construction of works such as side walls, pilings, breakwaters was analyzed, but due to their high costs and little effectiveness observed in other sections of the river, they were discarded. This research determined that a good way to protect this section is through the use of native plants of the sector such as Zygia Longifolia (Chiparo), whose roots, being abundant and long, form a true screen of protection against erosion, as evidenced with plants that are planted year after year. This research was carried out with the financial support of ARES from the Belgian Government.
\end{abstract}

Keywords: Pañacocha, erosion, Napo river, bank protection, native plants. 


\section{Introducción}

La forma de los cauces depende de las características hidrológicas, la geología, la vegetación, el clima y las actividades del hombre que afectan el perfil de los cauces naturales, su morfología es el resultado de la erosión, transporte y sedimentación de las partículas de suelo, su forma depende de las características hidrológicas y geológicas de la cuenca, y de las propiedades de los sedimentos. Los desplazamientos laterales en las curvas e incluso en tramos rectos que sufren los ríos especialmente en épocas de crecida, producen en la margen exterior o cóncava erosión, y en la interior o convexa sedimentación, procesos que deben ser controlados para evitar que el río afecte a las poblaciones e infraestructuras existentes, mediante la construcción de obras o el uso de materiales que fijen las orillas de forma permanente, tomando en consideración como trabajan estas estructuras para poder acoplarlas correctamente a las características propias del río. Además, se debe tomar en cuenta que el hombre es uno de los principales responsables de la inestabilidad de las orillas de un río debido a la deforestación, la agricultura, el transporte y otras actividades que lo alteran; conservar la vegetación propia de las riberas de los ríos es indispensable a la hora de proteger las márgenes de la acción erosiva de las aguas fluviales. Con estos antecedentes y con el fin de dar una solución a este problema que se viene produciendo constantemente en la parroquia de Pañacocha y sus embarcaderos, el Instituto de Excelencia en Transporte Intermodal y Fluvial, CETIF, de la Universidad Central del Ecuador, UCE, ha realizado una investigación para determinar cuáles son las principales causas por las que se produce este fenómeno con base a estudios hidrológicos, hidráulicos-batimétricos y de suelos.

\section{Método}

Para el estudio se realizaron in situ, levantamientos de información de las características del tramo en el río Napo, en la margen izquierda de la parroquia de Pañacocha y sus embarcaderos.

En una primera etapa, se recopiló información bibliográfica de la cuenca del río relacionada con la hidrología (caudales medidos en las estaciones hidrométricas más cercanas, a la zona en estudio, frecuencia de inundaciones, información de llu- vias, intensidad, velocidades y direcciones de corriente, tamaño y características de la cuenca del río Napo), e hidráulica, para determinar pendientes, análisis de palizadas, bancos de arena, formación de islotes y otras características hidráulicas.

En una segunda etapa, se levantó información de las características físicas importantes de las orillas del río, se hizo el levantamiento batimétrico del cauce, el calado del río se determinó mediante el uso de sondas batimétricas y de reglas limnimétricas establecidas. Se indagó a los pobladores para tener información histórica de la erosión de las orillas y desbordamientos del río.

En una tercera etapa se procesó la información recolectada utilizando softwares afines a esta investigación como: Google Earth (datos de GPS), HEC-RAS (Modelación del tramo en estudio), ARC-GIS (parámetros morfológicos) y AUTOCAD (topografía de la zona).

Por último, se analizaron las causas por las que se producen la erosión.

\subsection{Ubicación del estudio}

El estudio se realizó en la ribera izquierda del río Napo, Provincia de Sucumbíos, cantón Shushufindi en la parroquia de Pañacocha, en las coordenadas $0^{\circ} 26>22.67 » S ; 76^{\circ} 5>49.65 » \mathrm{O}$ aguas arriba, $y$ $0^{\circ} 28>6.46 » \mathrm{~S} ; 76^{\circ} 4>14.28 » \mathrm{O}$ aguas abajo.

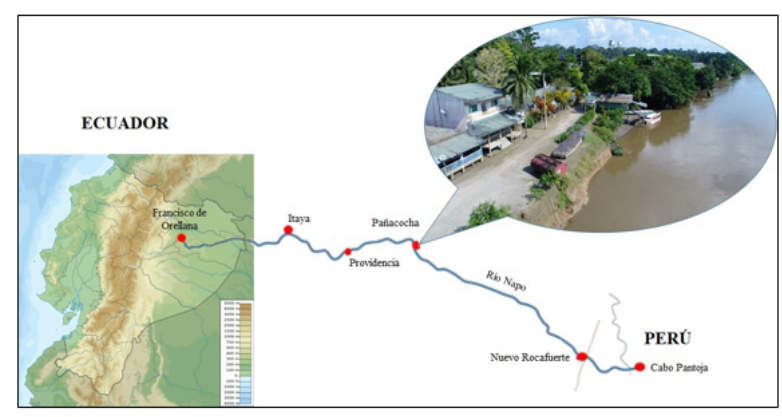

Figura 1. Ubicación del proyecto

La parroquia de Pañacocha se encuentra ubicada a $120 \mathrm{~km}$ aguas abajo de la ciudad de Francisco de Orellana (Coca), en la orilla izquierda del río Napo y a $409 \mathrm{~km}$ desde Quito. Tiene una extensión de 60.934,99 hectáreas, su territorio forma parte de la Reserva de Producción Faunística Cu- 
yabeno aportando con 59.000 hectáreas para su conformación. Esta parroquia es la menos poblada del cantón Shushufindi con una población de 860 personas [1].

\subsection{Problemática del sector}

La erosión en las orillas del río Napo, en el sector de Pañacocha ha ido incrementando paulatinamente con el paso de los años, haciendo que los pobladores de esta región vayan retrocediendo conforme la erosión de las orillas avanza, en vista de que sus viviendas se ven afectadas constantemente; además, debido al desbordamiento del río el agua se acumula en ciertos sectores produciendo malos olores y ayudando a la proliferación de mosquitos.

\subsection{Características generales del río Napo}

Según el estudio de factibilidad del dragado de los ríos Napo y Morona (Ecuador-Perú) mediante modelización matemática hidrosedimentológica. El lecho del río está conformado por arenas medias $(\mathrm{D} 10=0,2 \mathrm{~mm}, \mathrm{D} 50=0,35 \mathrm{~mm}, \mathrm{D} 90=0,6$ $\mathrm{mm}$ ), su pendiente media es del orden de $20 \mathrm{~cm} /$ $\mathrm{km}$ variando entre $13 \mathrm{~cm} / \mathrm{km}$ en los primeros 260 $\mathrm{km}$ desde la desembocadura, hasta $38 \mathrm{~cm} / \mathrm{km}$ en el extremo del área de estudio en Ecuador (Francisco de Orellana, $\mathrm{km} \mathrm{780)}$, su ancho es variable entre 400 y más de $2000 \mathrm{~m}$ (promedio del orden de $1000 \mathrm{~m}$ ), su caudal máximo es del orden de $6000 \mathrm{~m} 3 / \mathrm{s}$ en el tramo en estudio y el transporte sólidos oscila entre unas 1000 ton/día en estiaje a más de 200.000 ton/día en crecida. Es un río de tipo entrelazado e inestable con una intensa dinámica de formación de bancos de arena e islas.

Al ser un cauce inestable con altos valores de transporte de sedimentos en las zonas más profundas en condiciones de estiaje, que se generalizan a toda la sección en creciente, hace que estas zonas vayan variando en el tiempo, debido a su desequilibrio constante provocando erosión y sedimentación en diferentes sitios [2].

El río Napo al ser un río trenzado, con sinuosidades, presenta muchas curvas a lo largo de su recorrido, con erosión en la orilla exterior (cóncava) y sedimentación en la orilla interior (convexa).

Además, tiene una abundante carga de sedimentos de fondo y márgenes fácilmente erosionables que permiten la formación y el desarrollo de barreras en el cauce.

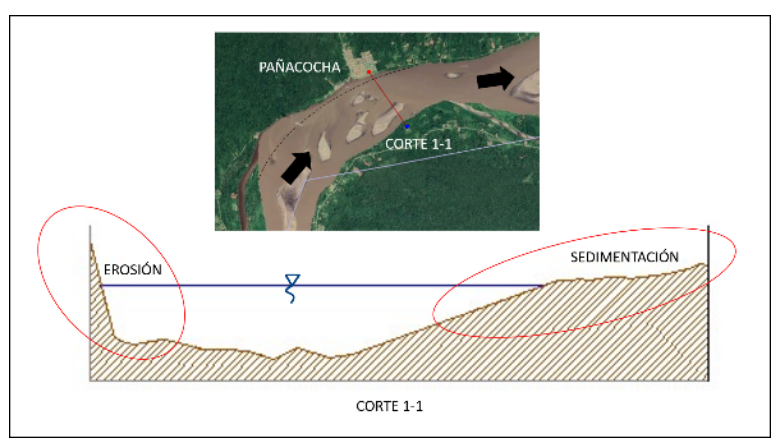

Figura 2. Erosión y sedimentación de una curva de un cauce fluvial

\subsection{Estudios realizados}

\subsubsection{Estudio hidrológico}

La hidrología tiene un papel fundamental en la planificación y uso de los recursos hídricos [3]. Para este proyecto los estudios hidrológicos de la cuenca del río Napo, proporcionaron información sobre su comportamiento, sus principales parámetros morfológicos, y el establecimiento de medidas de prevención ante posibles desastres naturales, como crecidas del río.

Los principales parámetros morfológicos que se determinaron en esta investigación son: la ubicación de la cuenca, cotas máximas y mínimas, el área, el perímetro, forma del cauce, el ancho promedio, el coeficiente de compacidad, el factor de forma, la relación de elongación [4]; para lo cual se utilizaron las siguientes ecuaciones.

Factor de forma de una Cuenca de Horton (F):

$$
F=\frac{A}{L^{2}}
$$

Dónde: $\mathrm{A}$ = Área del tramo de la cuenca

$$
\mathrm{L}=\text { Longitud de la cuenca }
$$

Relación de elongación (R):

$$
R=1.128 \frac{\sqrt{A}}{L}
$$




\section{Facultad de Ingeniería y Ciencias Aplicadas}

Dónde: $\mathrm{A}$ = Área del tramo de la cuenca

$\mathrm{L}=$ Longitud de la cuenca

Índice de Compacidad o Índice de Gravelious (K):

$$
K=0.28 \frac{P}{\sqrt{A}}
$$

Dónde: $\mathrm{P}=$ Perímetro del tramo de la cuenca

$$
\mathrm{A}=\text { Área del tramo de la cuenca }
$$

Tabla 1. Índice de Compacidad [5]

\begin{tabular}{ll}
\hline RANGO & DESCRIPCIÓN \\
\hline $1-1.25$ & Forma casi redonda a oval-redonda \\
$1.25-1.50$ & Forma oval-redonda a oval-alargada \\
$1.50-1.75$ & Forma oval-alargada a alargada \\
\hline
\end{tabular}

De la investigación realizada por Aguas Amazónicas en el año 2017, se tiene que el área de la cuenca del río Napo abarca aproximadamente 110.000 $\mathrm{km} 2$, representa el 1,6\% de toda la cuenca amazónica. El $60 \%$ de la cuenca está en Ecuador y el 40 $\%$ en Perú. Esta cuenca representa la mayor parte del área de drenaje del río Amazonas. La precipitación anual en la cuenca del Napo está entre los 2500 y $5000 \mathrm{~mm}$. La fluctuación anual del nivel del río es de aproximadamente $8,5 \mathrm{~m}$. Las llanuras a lo largo del río se inundan anualmente entre diciembre y mayo de cada año [6].

\subsubsection{Estudios hidráulicos-batimétricos}

Se realizaron dos batimetrías en diferentes épocas del año, de las que se obtuvieron los perfiles transversales y longitudinales del río, el área de los perfiles tipo, la velocidad y el caudal aproximado que pasa por una de las secciones transversales en el sector. Caudal que se utilizó para obtener las demás características fluviomorfológicas del tramo de estudio.

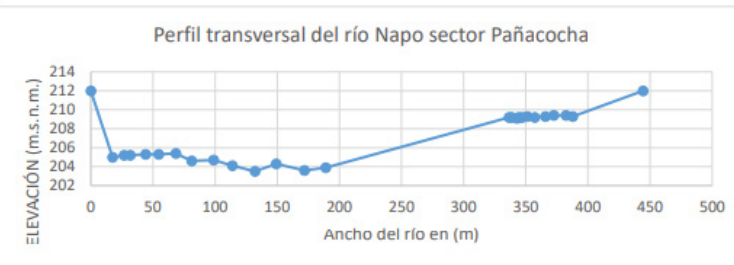

Figura 3. Perfil Transversal

\subsubsection{Estudio de suelos}

En la etapa de campo se recolectó una muestra de suelo a cielo abierto en la orilla izquierda del río en Pañacocha, con el fin de conocer sus características. El estudio se realizó en el Laboratorio de Mecánica de Suelos de la Facultad de Ingeniería y Ciencias Aplicadas de la UCE.

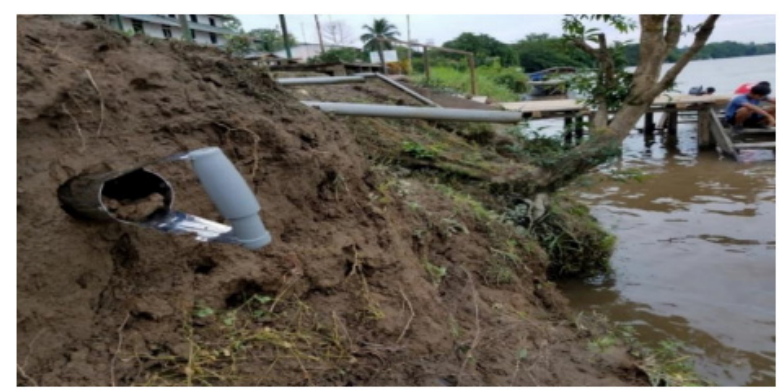

Figura 4. Características del suelo en Pañacocha

\section{Resultados y discusión}

\subsection{Características del tramo en estudio}

Con ayuda del software ARG-GIS se obtuvo el área, perímetro y pendientes de la cuenca y del río principal del tramo en estudio, información necesaria para calcular otros parámetros de forma, que se muestra en la siguiente tabla:

Tabla 2. Características del tramo en estudio en Pañacocha

\begin{tabular}{lll}
\hline Parámetro & Valor & Observaciones \\
\hline $\begin{array}{l}\text { Área del tramo de la cuenca del } \\
\text { río Napo (sitio del proyecto) }\end{array}$ & $25099.35 \mathrm{Km}^{2}$ & Software ARC - GIS \\
Coeficiente de compacidad K & $\mathrm{K}=1.68$ & $\begin{array}{l}\text { Mayor tendencia a las crecidas mientras sea próxi- } \\
\text { mo a 1 }\end{array}$
\end{tabular}


Factor de forma de la cuenca $\mathrm{F} \quad \mathrm{F}=0.33$

Relación de elongación $\mathrm{R}$

$\mathrm{R}=0.65$

Pendiente media del cauce So $=0.037 \%$ principal

Pendiente media del tramo de So $=9.89 \%$

la cuenca

Vegetación

Densidad de drenaje (Dd):
Ligeramente alargada

Pendiente media del tramo analizado, considerando $117 \mathrm{~km}$ aguas arriba y $110 \mathrm{~km}$ aguas abajo del lugar de estudio

Software ARC - GIS
Exuberante, encierra la mayor reserva forestal con gran variedad de especies

$$
\mathrm{Dd}=0.53 \mathrm{Km} / \mathrm{Km}^{2}
$$

$0.5 \mathrm{Km} / \mathrm{Km}^{2}$ Drenaje pobre, $3.5 \mathrm{Km} / \mathrm{km}^{2}$ es excepcionalmente bien drenada, por lo que el tramo de la cuenca es de drenaje pobre

Moderada
Además de las características del río, antes mencionadas, se determinó el coeficiente de rugosidad en el cauce principal mediante el método de Cowan [7], obteniendo la siguiente información:

Tabla 3. Valores para la estimación del coeficiente de rugosidad de Manning en el cauce principal del río

\begin{tabular}{llll}
\hline Factor ni & Condición del cauce & Calificación & valor \\
\hline no & $\begin{array}{l}\text { Material natural en } \\
\text { canal uniforme }\end{array}$ & $\begin{array}{l}\text { Arena de tama- } \\
\text { ño uniforme }\end{array}$ & 0.017 \\
n1 & $\begin{array}{l}\text { Irregularidad de la } \\
\text { superficie }\end{array}$ & Menor & 0.005 \\
n2 & $\begin{array}{l}\text { Variaciones en for- } \\
\text { ma y tamaño de la }\end{array}$ & 0.000 \\
& $\begin{array}{l}\text { sección transversal } \\
\text { n3 }\end{array}$ & $\begin{array}{l}\text { Presencia de Obs- } \\
\text { trucciones }\end{array}$ & \\
n4 & Vegetación presente & Baja & 0.005 \\
m5 & $\begin{array}{l}\text { Cantidad de Mean- } \\
\text { dros }\end{array}$ & Apreciable & 1.150 \\
& & Valor de “n” & 0.043 \\
\hline
\end{tabular}

De igual forma, mediante el método de Cowan, se determinó el coeficiente de rugosidad en las márgenes del río, información que se indica a continuación:

Tabla 4. Valores para la estimación del coeficiente de rugosidad de Manning en las márgenes de inundación

\begin{tabular}{llll}
\hline Factor ni & Condición del cauce & Calificación & valor \\
\hline no & $\begin{array}{l}\text { Material natural en } \\
\text { canal uniforme }\end{array}$ & $\begin{array}{l}\text { 20\% arena, } 80 \% \\
\text { finos }\end{array}$ & 0.013 \\
n1 & $\begin{array}{l}\text { Irregularidad de la } \\
\text { superficie }\end{array}$ & Menor & 0.001 \\
n2 & $\begin{array}{l}\text { Variaciones en for- } \\
\text { ma y tamaño de la } \\
\text { sección transversal }\end{array}$ & 0 \\
& $\begin{array}{l}\text { Presencia de Obs- } \\
\text { trucciones }\end{array}$ & \\
n3 & Vegetación presente & Mediano & 0.005 \\
n4 & $\begin{array}{l}\text { Cantidad de Mean- } \\
\text { dros }\end{array}$ & Apreciable & 1.15 \\
m5 & Valor de “n” & 0.031 \\
\hline
\end{tabular}


Otro método para determinar la rugosidad " $n$ " en el margen del río es mediante el ensayo de clasificación de suelos y su curva granulométrica.

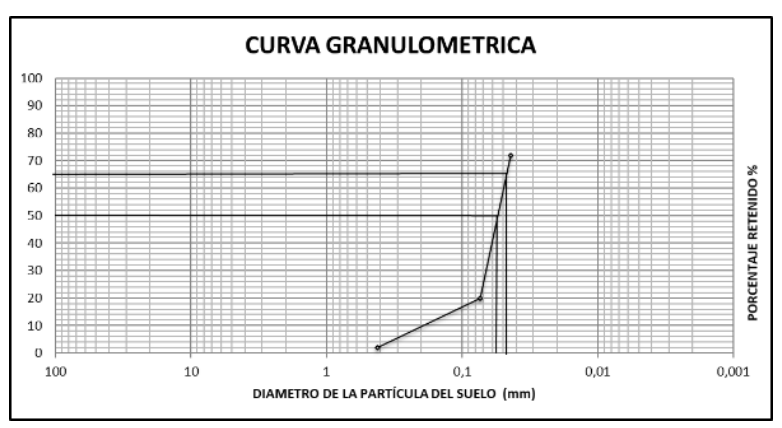

Figura 5. Curva granulométrica

Localizando los diámetros D65 y D50 en función de la partícula, información necesaria para el cálculo de la rugosidad " $n$ ", mediante las siguientes fórmulas [8]:

Raudkivi:

$$
n=0.0411 * D_{65}^{1 / 6}
$$

Simons y Senturk:

$$
n=0.0389 * D_{50}^{1 / 6}
$$

Garde \& Raju; Subramanya:

$$
n=0.047 * D_{50}^{1 / 6}
$$

Bray:

$$
n=0.0593 * D_{50}^{0.179}
$$

\begin{tabular}{|c|c|c|c|c|}
\hline \multicolumn{5}{|c|}{ Diámetros de la partícula } \\
\hline D65 = & 0.048 & $\mathrm{~mm}$ & & \\
\hline $\mathrm{D} 50=$ & 0.055 & $\mathrm{~mm}$ & & \\
\hline \multicolumn{3}{|c|}{ Raudkivi, 1976} & $n=0.0411 * D_{65}^{1 / 6}$ & 0.025 \\
\hline \multicolumn{3}{|c|}{ Simons y Senturk, 1976} & $n=0.0389 * D_{50}^{1 / 6}$ & 0.024 \\
\hline \multicolumn{3}{|c|}{$\begin{array}{l}\text { Garde \& Raju, 1978; } \\
\text { Subramanya } 1982\end{array}$} & $n=0.047 * D_{50}^{1 / 6}$ & 0.029 \\
\hline \multicolumn{3}{|c|}{ Bray, 1979} & $n=0.0593 * D_{50}^{0.179}$ & 0.035 \\
\hline \multicolumn{4}{|c|}{ Promedio $\mathrm{n}=$} & 0.028 \\
\hline
\end{tabular}

Obteniendo la información que se indica en la siguiente tabla:

Tabla 5. Fórmulas empíricas
El coeficiente de Manning determinado por el método de Cowan se utilizó en el cauce principal, mientras que para las márgenes se empleó el coeficiente promedio entre los dos métodos dando un valor de 0,03 aproximado.

Información requerida para la modelación en el software HEC-RAS, como se muestra en la siguiente tabla:

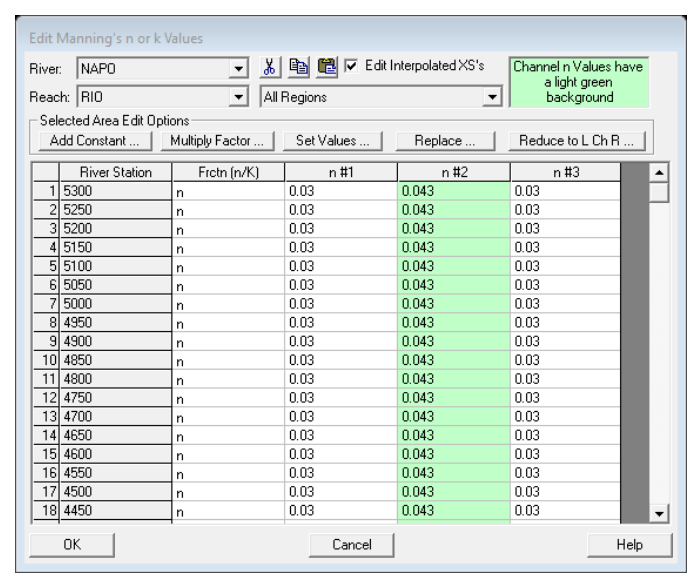

Figura 6. Coeficiente de rugosidad de Manning

Otro parámetro necesario para la modelación del río en HEC-RAS es la pendiente media del tramo en estudio. Información que se determinó con la diferencia de cotas entre Francisco de Orellana (El Coca) y Pañacocha, dividiendo para la distancia entre estos dos puntos.

La información que se muestra en la siguiente tabla fue calculada con los datos obtenidos en los viajes que el instituto ha realizado al sector en diferentes años.

Tabla 6. Pendiente media del tramo del río Napo sector Pañacocha

\begin{tabular}{ll}
\hline FECHA & PENDIENTE (\%) \\
\hline 27-jun-15 & 0.041 \\
19-nov-15 & 0.044 \\
27-oct-16 & 0.034 \\
28-ene-17 & 0.033 \\
Pendiente media $=$ & 0.038 \\
\hline
\end{tabular}


Debido a que en el sector no hay una estación hidrológica que proporcione información estadística de lluvias o caudales del Instituto Nacional de Meteorología e Hidrología, INAMHI, y que la información más cercana es de las estaciones del Coca o en Nuevo Rocafuerte, se optó, con ayuda del software CIVIL CAD 3D y fotos satelitales, determinar la topografía del sector de Pañacocha, con los que se obtuvo nuevos perfiles transversales y con ellos se calculó el área y el perímetro mojado del río en el sector; con esta información se calculó, la velocidad, el número de Froude, características hidráulicas necesarias para encontrar el caudal aproximado mediante la fórmula de Manning[7].

Tabla 7. Caudal aproximado que pasa por el sector de Pañacocha

\begin{tabular}{|c|c|c|c|c|}
\hline \multicolumn{5}{|c|}{ Caudal de un perfil transversal tipo en el río Napo - sector Pañacocha } \\
\hline DAT & & & \multirow{3}{*}{$Q=\frac{1}{n} A R_{h}^{3 / 4} S_{o}^{1 / 2}$} & \multirow{3}{*}{$\begin{array}{l}\text { dónde: } \\
\mathrm{Q}=\text { Caudal en } \mathrm{m} 3 / \mathrm{s}\end{array}$} \\
\hline$n=$ & 0.038 & & & \\
\hline$A=$ & 2368.85 & $\mathrm{~m} 2$ & & \\
\hline$P=$ & 445.97 & $\mathrm{~m}$ & & $\mathrm{n}=$ Rugosidad del río \\
\hline \multirow[t]{3}{*}{ So $=$} & 0.00035 & $\mathrm{~m} / \mathrm{m}$ & & $\mathrm{A}=$ Área del perfil $\mathrm{m} 2$ \\
\hline & & & $R_{h}=\frac{A}{P}$ & $\mathrm{P}=$ Perímetro mojado $\mathrm{m}$ \\
\hline & & & & $\mathrm{Rh}=$ Radio Hidráulico \\
\hline$Q=$ & 3550.39 & $\mathrm{~m} 3 / \mathrm{s}$ & & So $=$ pendiente del río \\
\hline$v=$ & 1.50 & $\mathrm{~m} / \mathrm{s}$ & & $\mathrm{v}=$ velocidad media $\mathrm{m} / \mathrm{s}$ \\
\hline
\end{tabular}

Incrementando el área y el perímetro mojado de cada perfil se obtuvo los caudales teóricos, necesarios para la modelación hidráulica en Pañacocha.

La cota de elevación en Pañacocha es 212 m.s.n.m. Cuando el río se desborda, sube aproximadamente $50 \mathrm{~cm}$, por lo tanto, la cota de inundación seria la 212,50 m s. n. m.; en estos y otros estados los caudales teóricos serían los que a continuación se indican:

Tabla 8. Caudales de crecida río Napo sector de Pañacocha

\begin{tabular}{llllll}
\hline \multicolumn{3}{l}{ Caudales de crecida en el río Napo sector Pañacocha } \\
\hline \multicolumn{3}{l}{ Elevación 210} & m.s.n.m. & \multicolumn{3}{c}{ Elevación 211} & m.s.n.m. \\
$\mathrm{n}=$ & 0.04 & & $\mathrm{n}=$ & 0.04 & \\
$\mathrm{~S}=$ & 0.00035 & $\mathrm{~m} / \mathrm{m}$ & $\mathrm{S}=$ & 0.00035 & $\mathrm{~m} / \mathrm{m}$ \\
$\mathrm{P}=$ & 445.97 & $\mathrm{~m} 2$ & $\mathrm{P}=$ & 988.97 & $\mathrm{~m} 2$ \\
$\mathrm{~A}=$ & 2368.85 & $\mathrm{~m} 2$ & $\mathrm{~A}=$ & 3349.85 & $\mathrm{~m} 2$ \\
$\mathrm{Q}=$ & 3372.87 & $\mathrm{~m} 3 / \mathrm{s}$ & $\mathrm{Q}=$ & 3533.68 & $\mathrm{~m} 3 / \mathrm{s}$
\end{tabular}

\begin{tabular}{llllll} 
Elevación 212 m.s.n.m. & \multicolumn{2}{l}{ Elevación 212,5 m.s.n.m. } \\
$\mathrm{n}=$ & 0.04 & & $\mathrm{n}=$ & 0.04 & \\
$\mathrm{~S}=$ & 0.00035 & $\mathrm{~m} / \mathrm{m}$ & $\mathrm{S}=$ & 0.00035 & $\mathrm{~m} / \mathrm{m}$ \\
$\mathrm{P}=$ & 990.97 & $\mathrm{~m} 2$ & $\mathrm{P}=$ & 991.97 & $\mathrm{~m} 2$ \\
$\mathrm{~A}=$ & 4330.85 & $\mathrm{~m} 2$ & $\mathrm{~A}=$ & 4821.35 & $\mathrm{~m} 2$ \\
$\mathrm{Q}=$ & 5414.47 & $\mathrm{~m} 3 / \mathrm{s}$ & $\mathrm{Q}=$ & 6470.28 & $\mathrm{~m} 3 / \mathrm{s}$ \\
\hline
\end{tabular}

\subsection{Modelación del tramo del río Napo sector Pañacocha}

La modelación del río se realizó con los softwares ARC-GIS, HEC-RAS y CIVIL CAD 3D en un tramo aproximado de $5 \mathrm{~km}$, utilizando la siguiente información: perfiles longitudinales, perfiles transversales, caudales, pendiente hidráulica del río, rugosidad y velocidades.

En el siguiente gráfico, se observa la inundación en el sector de Pañacocha, cuando el río supera $\operatorname{los} 6000 \mathrm{~m}^{3} / \mathrm{s}$ 


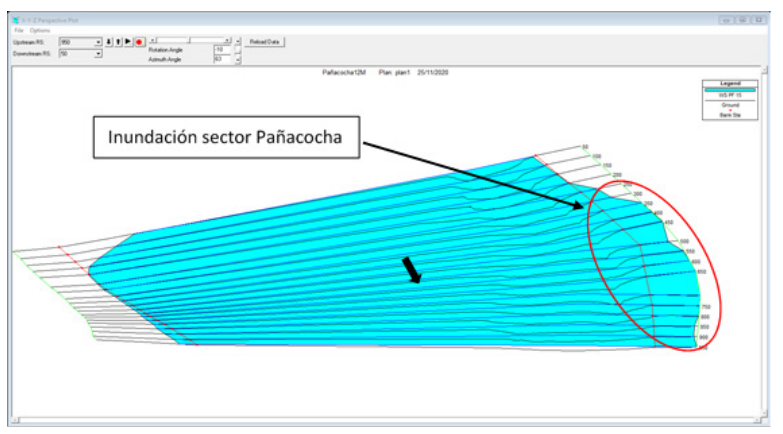

Figura 7. Inundación en Pañacocha y sus embarcaderos

Como resultado de la modelación del río con su caudal máximo calculado, a continuación, en el gráfico se observa que el sector de Pañacocha se inunda, provocando que las orillas se erosionen, cuando el nivel del agua baja regresando a su caudal medio.

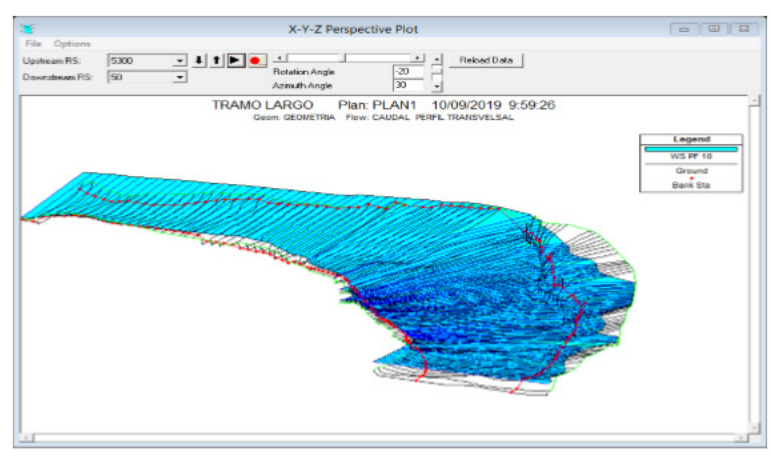

Figura 8. Modelación del tramo en estudio en Pañacocha

\subsection{Causas por las que se produce la erosión}

Los procesos de erosión en los ríos son muy complejos y obedecen a fenómenos hidráulicos y geomorfológicos, en los cuales interviene una gran cantidad de variables como: la profundidad del cauce, la inestabilidad lateral de la corriente, transporte de sedimentos, y de material flotante, las olas producidas por la velocidad con la que circulan las embarcaciones, los deslizamientos en las riberas de las corrientes, dinámica fluvial, los efectos de la intervención antrópica y las velocidades propias del río, sobre todo en crecidas. En el estudio se determinó que existen algunas causas por las que se produce la erosión en las orillas del río Napo en el sector de Pañacocha, como:

\subsubsection{Inundaciones o grandes crecidas produci- das por intensas precipitaciones.}

De la modelación con HEC-RAS se determinó que una de las causas por las que se produce la erosión en las orillas del río Napo y sus embarcaderos es debido a las intensas precipitaciones que se presentan en la zona, las mismas que causan inundaciones o desbordamientos. La orilla izquierda del río sufre un proceso de intensa erosión hídrica, por el desborde del caudal de crecida y luego por el retorno de este caudal a su cauce [9].

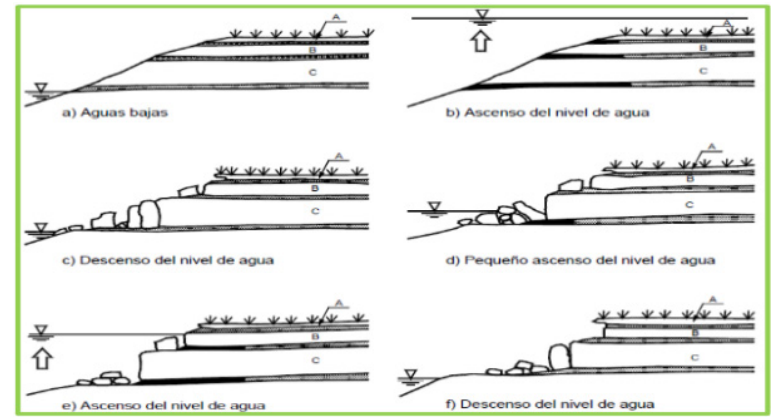

Figura 9. Proceso de erosión de las orillas de un río por descenso del nivel de agua después de una crecida.

\subsubsection{Tránsito de embarcaciones que circulan a gran velocidad}

Las altas velocidades con que transitan los «deslizadores» al momento de pasar por el sector, producen grandes olas que chocan contra las orillas del río debilitándolas y produciendo que éstas erosionen. El impacto del agua sumado a la abrasión de partículas, sedimentos, arena transportadas en el vaivén de las olas, van desgastando las orillas [9].

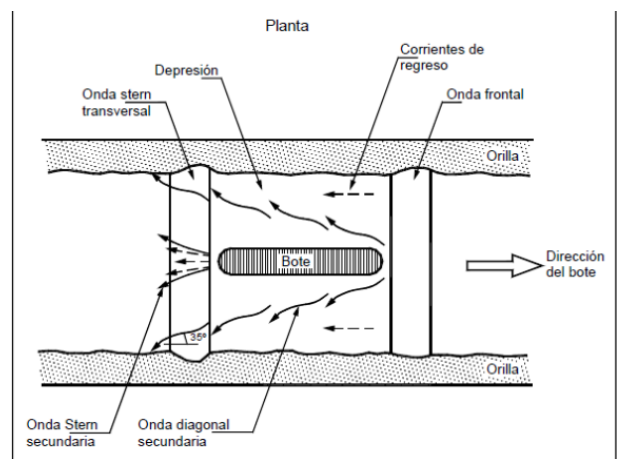

Figura 10. Olas producidas por el tránsito de embarcaciones 


\subsubsection{Velocidad del flujo del río}

La velocidad del río es otra de las causas por la que se produce erosión en las orillas del río Napo, la razón es que Pañacocha se encuentra aguas abajo de una curva donde la velocidad del río aumenta, provocando el típico fenómeno de erosión en la parte interna de la curva.

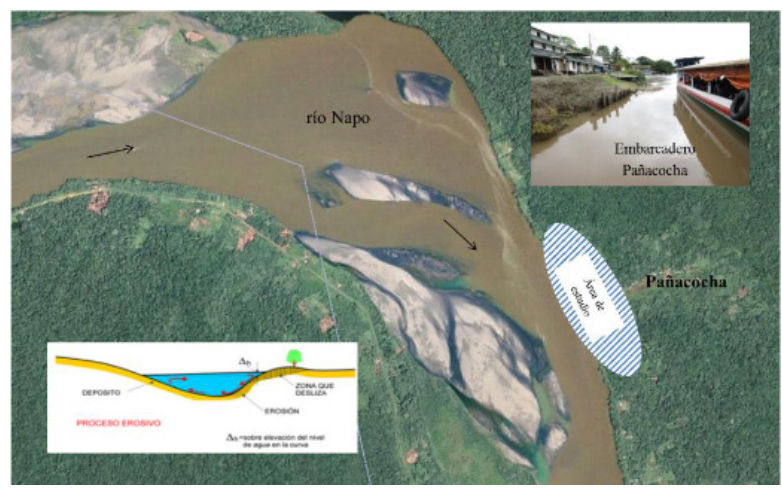

Figura 11. Curva del río Napo aguas arriba del sector Pañacocha

\subsubsection{Tipo de suelo presente en la zona}

Del estudio de mecánica de suelos se determinó que es un Limo (ML) de poca resistencia y plasticidad por lo que, al encontrarse desprotegido, las gotas de lluvia impactan en el suelo fuertemente rompiendo su estructura, fenómeno conocido como erosión por salpicadura, las partículas y sedimentos «salpican» una cierta altura, luego caen y tapan los poros naturales de la tierra por donde filtra y absorbe agua, y al incrementarse el flujo se lava la superficie [9].

\section{Caída de agua}

Golpe de agua

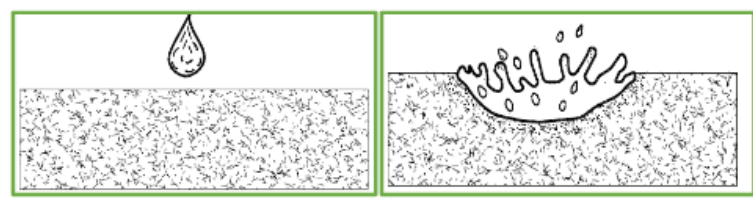

Desprendimiento

Esparcimiento
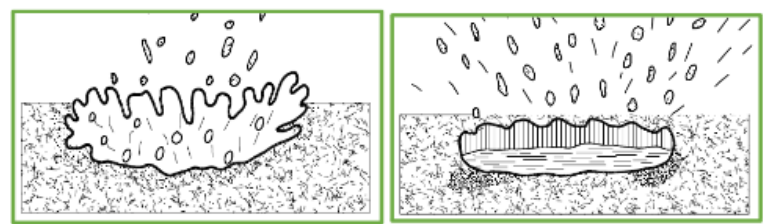

Figura 12. Erosión producida por lluvia

\subsubsection{Otras causas}

Existen otros factores que en menor grado erosionan el suelo del sector como la deforestación y vientos fuertes.

\subsection{Alternativas de solución}

Para proteger las márgenes de un río se tienen varias alternativas, como: recubrimientos, muros, enrocados, espigones, tablestacado, pilotajes, barreras con sacos de arena, uso de plantas nativas, entre otros; el utilizarlos depende de las características del sector, criterios técnicos, ambientales y los costos para su implementación.

Para el estudio, de acuerdo a los criterios técnicos antes mencionados, la mejor manera de proteger este sector es mediante el uso de plantas nativas.

\subsubsection{Protección de márgenes mediante el uso de plantas nativas}

Algunas plantas nativas eficaces para proteger márgenes de río, son las siguientes: Chiparo (Zygia longifolia), Sauce llorón (Salix babilonica), Fresno (Fraxinus viridis) y Alamo [10].

Estas plantas nativas se adhieren fuertemente con sus abundantes raíces, evitando que el suelo se desprenda cuando se producen crecientes y decrecientes.

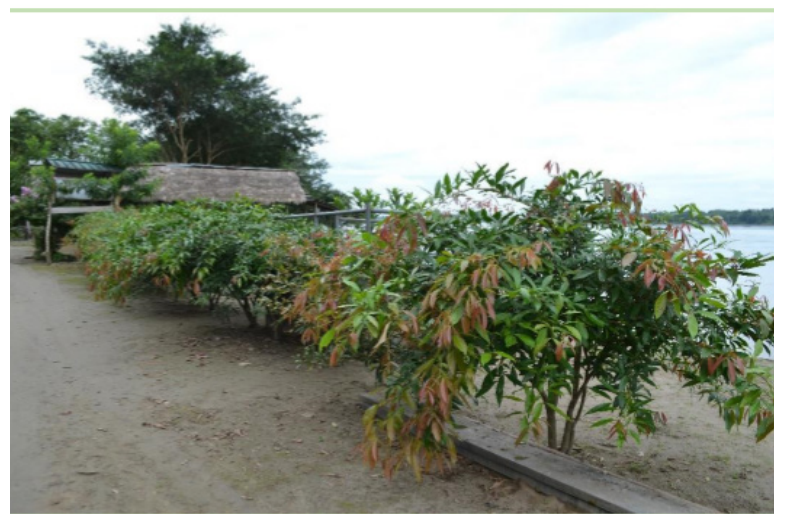

Figura 13. Plantaciones de Chíparo en Pañacocha para la protección de su ribera

\subsubsection{Siembra de árboles en la ribera}

La reforestación en el tramo de estudio se está realizando mediante la plantación de árboles de Chíparo en franjas longitudinales en las orillas del río, en distancias de alrededor de 2 metros entre 
sí, para proteger la población de Pañacocha y sus embarcaderos.

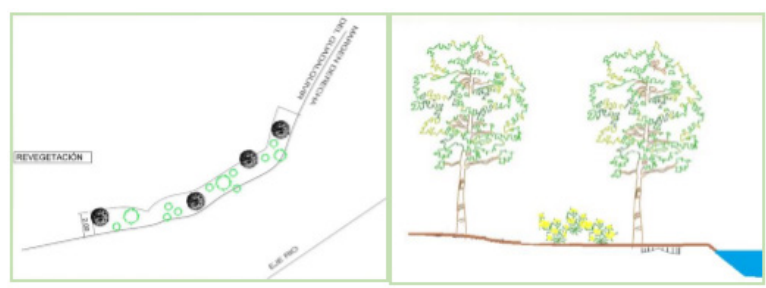

Figura 14. Reforestación con árboles de Chíparo

\subsubsection{Función de la vegetación}

La vegetación presenta resistencia al flujo y al transporte de sedimentos, su efecto más importante en los ríos es proteger el suelo contra la erosión de impacto, brindando rugosidad al terreno y reduciendo la velocidad erosiva [11].

Mientras más densa y homogénea sea la cubierta vegetal, mayor es su efectividad en la disminución de la erosión laminar, reduciendo significativamente la velocidad del viento, quitándole energía que erosiona la capa superficial [11].

Las raíces profundas del chíparo previenen el movimiento de masas en pendientes, debido a que se encuentran fuertemente ancladas, mientras que las superficiales, que son finas, ayudan a sujetar el suelo, formando un conglomerado firme y difícil de romper. Por estas razones, la siembra de estas plantas es una forma efectiva de controlar la erosión [12].

Las raíces de las plantas ayudan a sostener el suelo en su lugar, siendo las raíces superficiales (A) las más adecuadas para controlar la erosión hídrica y eólica. Las raíces más profundas ( $\mathrm{B}$ y $\mathrm{C}$ ) ayudan a prevenir movimientos de masas en pendientes [11].

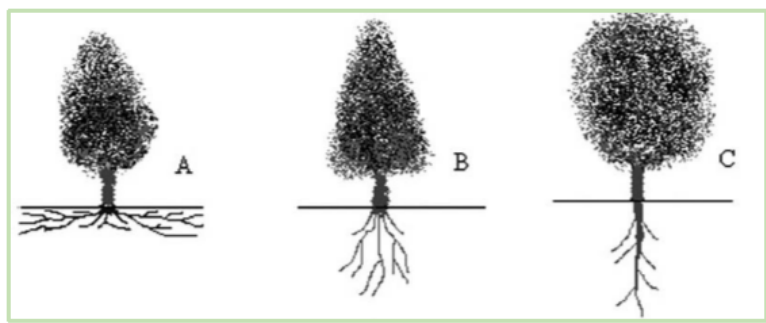

Figura 15. Diferentes tipos de raíces que ayudan a sostener el suelo

\subsection{Impactos por erosión y desbordamiento del río Napo}

Los flujos laterales y helicoidales son muy peligrosos porque socavan y desbordan las orillas del río produciendo un deterioro ambiental por erosión y haciendo que las personas se desplacen a lugares más seguros [13].

Los principales impactos que se tienen en Pañacocha son: inundación, contaminación del entorno de la población, pérdida de especies naturales y animales, entre otros.

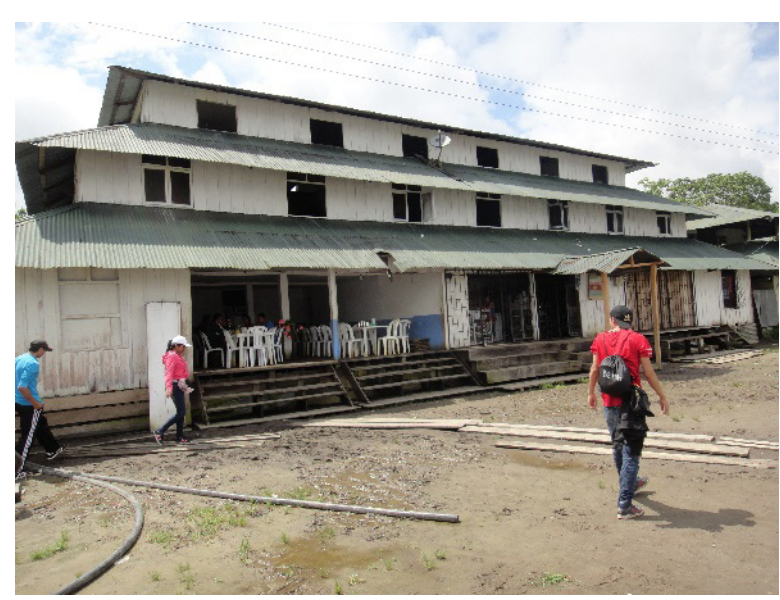

Figura 16. Piso de viviendas sobre elevadas para evitar la inundación de agua y sedimentos

\section{Conclusiones}

- Se evidenció que las causas de la erosión en Pañacocha son debido a: grandes precipitaciones del río, altas velocidades del agua con flujo tangencial, oleaje producido por las embarcaciones que transitan a grandes velocidades, y las características propias del suelo.

- Una buena alternativa para controlar la erosión en la ribera de Pañacocha, por su efectividad y bajo costo es, la siembra del Chiparo, árbol nativo del sector cuyas raíces forman una malla que ayudan a sostener el suelo.

- Mientras más densa y homogénea sea la cubierta vegetal, mayor será su efectividad en la disminución de la erosión, las raíces profundas anclan el suelo previniendo su movimiento, las raíces superficiales finas sujetan el suelo, formando un conglomerado firme y difícil de romper. 
- Cualquier método efectivo para controlar la erosión en la ribera de un cauce fluvial, debe ser elegido en función de las características del sector, del proceso erosivo, y, tomando en consideración los factores técnicos, ambientales y económicos.

- La erosión de las orillas del río Napo en Pañacocha causa el incremento de sedimentos y la turbiedad del agua afectando a las especies acuáticas.

- Las crecidas y desbordamientos causan encharcamiento, presencia de insectos, malos olores, ensanchamiento del rio, desplazamiento de la población y otros problemas ambientales.

- Es recomendable llevar una estadística de la siembra y desarrollo de Chípalo.

- Se recomienda difundir esta investigación en la Amazonia.

- Dar charlas técnicas a las personas del sector y de otros sectores sobre la problemática de la erosión, el cómo prevenirla y controlarla con la conservación y siembra de vegetación propia del lugar.

- Para estabilizar la erosión en Pañacocha se deben adoptar métodos que provoquen el menor impacto paisajístico y sean compatibles con las características ambientales y económicas del sector.

- Realizar estudios a nivel de proyecto definitivo considerando la parte económica y el entorno ambiental.

\section{Referencias}

[1] Instituto Nacional de Estadísticas y Censos-INEC (2010). Base de datos de población y vivienda parroquia de Pañacocha.

[2] Cardini, J., Garea, M., Legal, N., Faya, T., Reguero, G., Suarez, A. y Campos, M. (2015). Factibilidad del Dragado de los Ríos Napo y Morona (Ecuador-Perú) mediante Modelización Matemática Hidrosedimentológica. Serman \& Asociados S. A., jun.

[3] Recursos Hidráulicos. (2014). Recursos Hidráulicos. Obtenido de http://julianrojo. weebly.com/hidrologiacutea.html\#: :tex$\mathrm{t}=$ En\%20la\%20actualidad\%20la\%20hidrolo-
g\%C3\%ADa,la\%20acci\%C3\%B3n\%20de\%20
r\%C3\%ADos\%20y

[4] Jiménez Escobar, H. (1986). Hidrología Básica 1. Cali, Colombia: Facultad de Ingeniería, Universidad del Valle.

[5] Maidment, D. R. (1993). Handbook of hydrology (Vol. 9780070, p. 397323). New York: McGraw-Hill.

[6] Society, 2. W. (2017). Aguas amazónicas. Obtenido de http://aguasamazonicas.org/cuencas/principales-sub-cuencas/napo/

[7] Chow, V. (1983). Hidráulica de los canales abiertos. México: DIANA, S. A. - Roberto Gayol 1219, Esq. Tlacoquemécatl, Mexico 12 D. F.

[8] Espinoza Girón, P. y Lazo Quevedo, J. (2012). Estudio para Optimización de la Conducción Labrado-Tuñi en la Central Saucay del Complejo De Generación Hidroeléctrica Machángara. Obtenido de http://dspace. ucuenca.edu.ec/bitstream/123456789/773/1/ ti899.pdf

[9] Suárez Díaz, J. (2001). Control de Erosión en Zonas Tropicales. Bucaramanga-Colombia: División Editorial y de Publicaciones de la Universidad Industrial de Santander.

[10] Valdés, A. (2010). Cómo controlan la erosión las raíces de las plantas. Divulgación Científica y Tecnológica de la Universidad Veracruzana, 7-9.

[11] phi - LAC. (2010). Programa Hidrológico Internacional de la UNESCO para América Latina y el Caribe. Obtenido de Procesos de erosión - sedimentación en cauces y cuencas: http://eias.utalca.cl/isi/publicaciones/erosion_y_sedimentacion_vol1.pdf

[12] Méndez, C. (6 de abril de 2017). Chiparo, el árbol que salvó a un barrio de la tragedia en Mocoa. Obtenido de Mongabay Latam, periodismo ambiental independiente: ht- 
tps://es.mongabay.com/2017/04/chiparo-arbol-salvo-barrio-la-tragedia-mocoa/
[13] Alvarado Ancieta , C. A. (1 de Marzo de 2013). SciELO México. Obtenido de http:// www.scielo.org.mx/pdf/tca/v4n1/v4n1a3.pdf 